\title{
MUJERES MAYORES Y ACTIVIDAD FÍSICA
}

\author{
JUAN TORTOSA MARTÍNEZ \\ y LILYAN VEGA RAMÍREZ \\ Universidad de Alicante
}

Recibido: 21/01/2013

Aceptado: 27/03/2013

\section{Resumen}

La igualdad de la mujer es un tema que se aborda de diferentes perspectivas siendo la actividad física uno más de ellos. En el siguiente artículo haremos un análisis y reflexión del papel histórico que ha tenido la mujer en el campo de la actividad física, analizar desde una perspectiva de género la participación en actividades físicas de las personas mayores, así como su relación con diferentes variables asociadas a la salud general, la memoria o la salud emocional. El artículo se centra en un análisis de percepción de estilos de vida saludable de una muestra española de 300 personas mayores, llevado a cabo en la primera fase del proyecto de investigación europeo Empowering Health Learning for the Elderly (EHLE).

Palabras clave: actividad física, género, personas mayores, estilos de vida saludables, EHLE.

\begin{abstract}
The equality of women is a topic that may be approached from different perspectives, and physical activity is one of them. In this article, an analysis and reflection of the historical role that women have played in the physical activity field is conducted, and the participation of older adults from a gender perspective is analyzed, as well as the relationship between physical activity and different health related variables such as memory or emotional health. The article is focused on a perception analysis of healthy lifestyles conducted in a sample of 300 Spanish older adults, as a part of the European research project named Empowering Health Learning for the Elderly (EHLE).
\end{abstract}

Keywords: physical activity, gender, older adults, healthy lifestyles, EHLE. 



\section{Introducción}

El tema de la igualdad de la mujer, es un tema que se viene abordando en los últimos años desde diferentes perspectivas, sobre todo en el plano social y cultural. La actividad física es un componente más que está inmerso en el quehacer cotidiano por lo que no puede excluirse de su análisis y reflexión.

Si nos remontamos a los inicios de la humanidad, el papel de la mujer ha sido secundario, basado en el principio de que las mujeres son diferentes a los hombres, inferiores y dependientes ${ }^{1}$. Antiguos escritos de griegos romanos y hebreos muestran el sometimiento de la mujer ante los hombres².

La biología intenta explicar las diferencias entre hombres y mujeres para dar una explicación del pasado de subordinación de las mujeres en muchas culturas y sociedades humanas. Anderson y Ziusser ${ }^{3}$, argumentan que la biología distingue diversas diferencias fisiológicas entre las mujeres y los hombres. Si las condiciones de dieta, salud y ejercicio son iguales, las mujeres serán, en promedio, un 10\% más bajas, más ligeras y débiles que los hombres. Los hombres producen mayores niveles de testosterona, que se ha asociado con una disposición diferente para responder a la agresividad. Las mujeres tienen la capacidad de dar a luz y de vivir más que los hombres, lo cual algunos asocian a la mayor producción de estrógeno en las mujeres. Sin embargo, es obvio que una constante (la biología) no puede explicar una variable (las diferencias de una sociedad a otra en lo que respecta al papel asignado a la mujer $)^{4}$.

Este tipo de estereotipo de mujer pasiva, no agresiva y débil, cuyo principal papel era la maternidad y el cuidado de la familia se mantuvo por siglos, perjudicando a las mujeres en la participación de la práctica deportiva, ya que ésta se consideraba competitiva y agresiva, y por tanto actividad propia de los

1. Garcia Ferrando, Manuel. Aspectos sociales del deporte: una reflexión sociológica. Madrid, Alianza Editorial, 1990.

2. ANDERSON, Bonnie y ZIUSSER, Judith. Historias de las mujeres: una historia propia. Barcelona, Crítica, 2003.

3. Ibidem.

4. MEAD, Margaret, Sexo y temperamento en tres sociedades primitivas. Barcelona: Paidós Ibérica, 2006. 
hombres. García Ferrando ${ }^{5}$ atribuye este hecho a la sociedad griega, en la que se crearon los juegos olímpicos, ya que la mitología dominante atribuía a los dioses las cualidades de actividad, vigor y fuerza y a las diosas los rasgos de belleza, sexualidad y pasividad.

La actividad física y el deporte de las mujeres han estado relegados a un segundo plano desde la antigüedad, donde solo podían participar en los Juegos de Hera griegos, y que se celebraban después de los Juegos Olímpicos. Pierre de Coubertin, al reinstaurar los Juegos Olímpicos modernos, excluyó a la mujer de los mismos y le reservó el «honor» de «coronar al vencedor». Para que la mujer pudiera participar en el deporte moderno debía asumir el estereotipo hegemónico de la feminidad. Inicialmente, este estereotipo se limitaba a determinadas modalidades. Así, en los juegos de 1900, la mujer fue admitida en algunas pruebas: golf, tenis, tiro con arco, vela y patinaje $e^{6}$.

A partir de la II Guerra Mundial los movimientos feministas promovieron que las mujeres pudieran participar en otros deportes, asumiendo roles hasta entonces propios del género masculino. Este hecho provocó que en muchos casos sufrieran discriminación social. Las atletas eran tachadas de «marimachos» si participaban en pruebas consideradas de «hombres» ${ }^{7}$. A partir de los años 70 la imagen y rol de la mujer fue cambiando, favoreciendo el concepto de la mujer deportista ${ }^{8}$.

Hoy la práctica deportiva femenina está mucho más generalizada tanto en la práctica deportiva de alto rendimiento como en actividades recreativas de ocio o actividades físicas de salud, estando la presencia de la mujer en estos ámbitos más en consonancia con los avances sociales?. Sin embargo, el deporte es posiblemente uno de los ámbitos donde más se ve todavía al hombre como superior ${ }^{10}$. Queda mucho camino por recorrer para que las mujeres de todas las edades disfruten de igualdad de oportunidades respecto a los hombres en el ámbito deportivo. En concreto, las mujeres mayores españolas han

5. Garcia Ferrando, Manuel, Op. Cit., p. 1.

6. LOPEZ, Cristina. y ALVARIÑAS, Miriam. "Análisis muestrales desde una perspectiva de género en revistas de investigación de Ciencias de la Actividad Física y del Deporte españolas». Apunts: Educación física y deportes, 106 (2011), pp. 62-70.

7. MINISTERIO DE ASUNTOS SOCIALES. Mujer y deporte. Barcelona: Ministerio de Asuntos Sociales, 1990.

8. Lopez, Cristina. y Alvariñas, Miriam. Op. Cit., p. 3.

9. MERINO, Antonio. Mujer y deporte. Fórmula de integración en la sociedad actual. Motivaciones y necesidades específicas de la mujer en la realización de la práctica físico-deportiva. Diputación provincial de Jaén, 2007. ttp://issuu.com/amerinomandly/docs/integracion_ 20social_20act_20fisica_20mujer.

10. BERNSTEIN, Alina. «Is it time for a victory lap? Changes in the media coverage of women in sport». International Review for the Sociology of Sport 37 (2002), pp. 415-428. 
sido educadas sin acceso a la educación física en la mayoría de los casos, y con unos marcados estereotipos que relegaban a la mujer a tener muy pocas oportunidades de acceso al deporte. Sin duda, sus niveles de actividad física actual vienen determinados por esta educación.

Por otro lado, en las últimas décadas, se han venido produciendo cambios demográficos significativos en Europa, donde los mayores de 65 años representan hoy el $16,9 \%$ de la población y se situarán por encima del $30 \%$ en el $2025^{11}$. Esto supone un desafío para todos los países europeos, y requieren la puesta en práctica cada vez más urgente de políticas de «envejecimiento activo» centradas en la promoción de la vida autónoma de los ciudadanos antes y después de la jubilación. La población española será la más anciana de la UE en 2050, con un porcentaje del 35,6\% de mayores de 65 años ${ }^{12}$. Según los datos del INE en el 2008, la población femenina mayor a 60 años es superior a la masculina y este porcentaje aumenta a medida que aumenta la edad.

La disminución en la producción de estrógenos que se produce en la mujer adulta mayor, hace que el envejecimiento femenino se produzca a ritmos distintos que están relacionados con factores hereditarios y ambientales ${ }^{13}$. Uno de los principales factores de riesgo de mortalidad y de numerosas patologías asociadas al envejecimiento en la mujer contemporánea son los hábitos de vida sedentaria ${ }^{14}$.

Por el contrario, el ejercicio físico regular y supervisado puede prevenir procesos y alteraciones que se asocian al climaterio, como hipertensión, mala regulación de la glucosa, sarcopenia, osteoporosis, hipercolesterolemia y obesidad $^{15}$.

La comisión Europea designó el año 2012, como el año del envejecimiento activo, con el fin de mejorar los hábitos saludables y llevar una vida más activa. La actividad física para conseguir estilos de vida saludables es un factor clave en la promoción de la salud y la calidad de vida entre los mayores. Lher

11. EHLE - Empowering Health Learning for the Elderly. Funding: 134023 - LLP - 1 2008 -1 - IT - GRUNDTVIG-GMP http://www.ehle-project.eu/

12. IMSERSO. Libro blanco del envejecimiento activo. Madrid, 2011.

13. SEMI. Protocolos Osteoporosis. Sociedad Española de Medicina Interna, 2004.

14. ORGANIZACIÓN MUNDIAL DE LA SALUD. Health and Development Through Physical Activity and Sport, http://whqlibdoc.who.int/hq/2003/WHONMH_NPH_PAH_03.2.p., consultado el 6-12-2012.

15. ORGANIZACIÓN MUNDIAL DE LA SALUD. Recomendaciones mundiales sobre actividad física para la salud, http://www.who.int/dietphysicalactivity/publications/9789241599979/es/index.html consultado el 20-11-2012. 
y Thomas ${ }^{16}$ mencionan diversos estudios (Havighurst; Lemon y Cols; Longino y cols) que demostraron que las personas que se mantienen activas formal o informalmente disfrutan de un mayor gozo por vivir, se adaptan mejor y viven más años. Según datos aportados por la OMS en el 2010, un 50\% de las discapacidades en los países europeos se podrían evitar con medidas de prevención apropiadas, incluyendo la actividad física en estas medidas.

En este contexto, la Universidad de Alicante participó en un proyecto europeo denominado Empowering Health Learning for Elderly (EHLE) Project, financiado por la Comisión Europea dentro del programa Lifelong Learning, acción Grundtvig, y que fue llevado a cabo conjuntamente por cuatro socios de diferentes países europeos (Francia, Holanda, Italia y España). El objetivo final del proyecto era mejorar las competencias en estilos de vida saludable de las personas mayores y las destrezas y competencias de los profesionales que trabajan con las personas mayores.

El proyecto se desarrolló en varias fases. La primera fase del proyecto consistió en realizar un análisis específico por países de la percepción que las personas mayores tenían sobre los mensajes relacionados con los estilos de vida saludables. Las conclusiones extraídas de este análisis sirvieron para detectar las necesidades y lagunas a subsanar respecto a estilos de vida saludable de las personas mayores, y así diseñar los materiales didácticos en una segunda fase.

El presente artículo se centra en el análisis de la encuesta de percepción de estilos de vida saludable de la muestra española, llevado a cabo en la primera fase del proyecto.

El objetivo de esta investigación es, por tanto, teniendo en cuenta el papel histórico que ha tenido la mujer en el campo de la actividad física, analizar desde una perspectiva de género la participación en actividades físicas de las personas mayores, así como su relación con diferentes variables asociadas a la salud general, la memoria o la salud emocional.

\section{Metodología}

Los datos de esta investigación forman parte de un estudio descriptivo transversal más amplio incluido en el proyecto europeo EHLE. En este proyecto, para realizar el estudio de la percepción de las personas mayores sobre sus estilos de vida saludable, se entrevistó a 300 personas de entre 55 y 74 años en cada uno de los 4 países participantes $(n=1200)$. El instrumento utilizado fue un cuestionario de preguntas cerradas que incluía 64 ítems sobre estilos

16. LeHR, Ursula. y ThOMAe, Hans. Psicología de la senectud: proceso y aprendizaje del envejecimiento. Barcelona, Herder, 2003. 
de vida como la actividad física, la dieta, el alcohol y el tabaco, el uso de medicamentos, la memoria y la salud emocional.

Los datos que se presentan en este artículo son referidos al cuestionario de salud percibida de la muestra española $(n=300)$, centrando el análisis, fundamentalmente, en las variables relacionadas con la actividad física, la condición física y la salud percibida de la muestra desde una perspectiva de género.

La muestra española fue seleccionada mediante un sistema intencional y no probabilístico de entre los alumnos de la Universidad Permanente de la Universidad de Alicante de entre 55 y 85 años. La Universidad Permanente ofrece cursos de formación a personas mayores de 50 años.

\subsection{Análisis estadístico}

Los datos fueron analizados mediante el paquete estadístico SPSS-15 para las Ciencias Sociales. Se emplearon estadísticos descriptivos como porcentajes y medias para describir la situación percibida de la muestra respecto a las variables de estudio.

Para valorar el uso de estadísticos paramétricos o no paramétricos a la hora de realizar correlaciones y diferencias de medias, se realizó la prueba de normalidad Kolmogorov-Smirnov, y en los casos necesarios el test de Levene para comprobar la homogeneidad de varianzas.

Para determinar la correlación entre la condición física percibida y los niveles de actividad física con variables relacionadas con la salud percibida, el estado de ánimo, y la memoria, se utilizó el coeficiente de correlación de Pearson para aquellas variables que cumplían criterios de normalidad, y Spearman para aquellas variables que no las cumplían.

Las diferencias de género respecto a las variables de estudio se analizaron de forma descriptiva mediante porcentajes y medias, y empleando la prueba $t$ de Student para comparar medias de muestras independientes. El test de Chicuadrado se empleó para las correlaciones con variables categóricas. El nivel de significación se estableció en p<0,05 bilateral.

\section{Resultados}

El total de la muestra analizada en este estudio es de 300 personas de las cuales el 38\% corresponde al sexo masculino y $62 \%$ del sexo femenino. El 49\% de las personas encuestadas tiene entre 55 y 64 años, el $37 \%$ entre 65 y 74 . El nivel socioeconómico y de educación de los participantes es medio-alto. 
Tabla 1. Distribución de la muestra por sexo

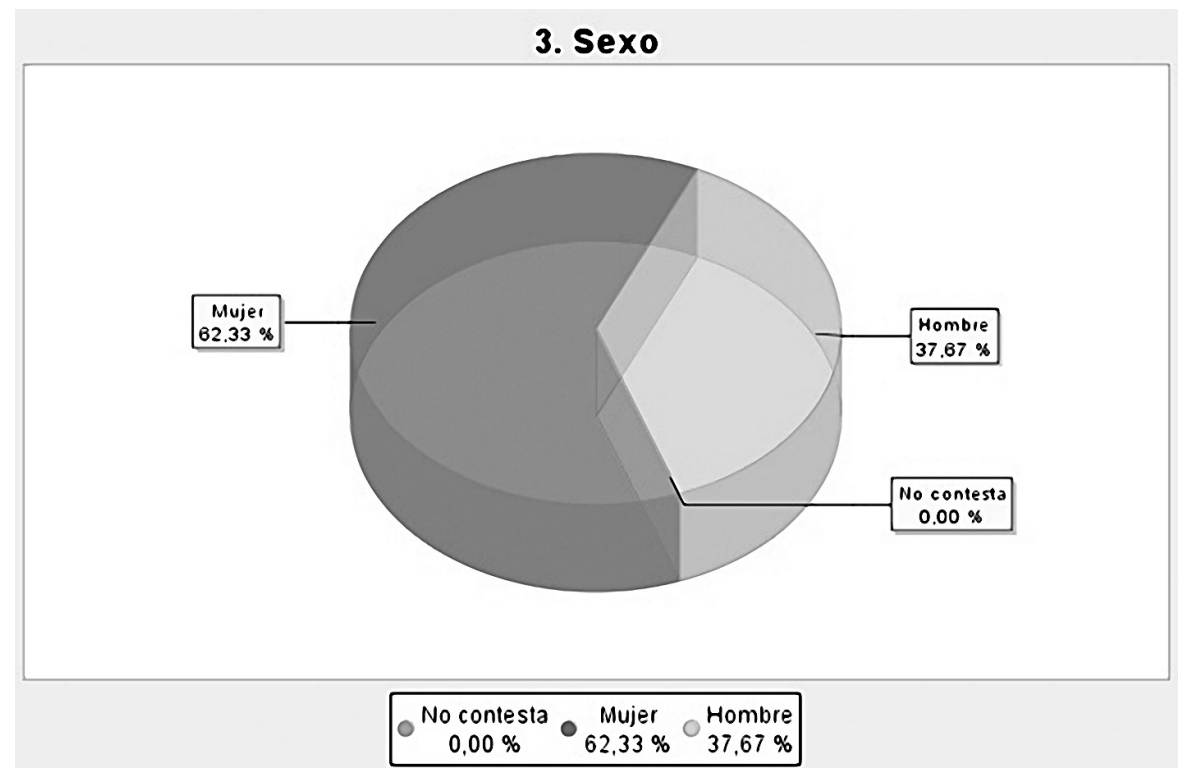

\subsection{Actividad física y condición física}

El 55,67\% de las personas participantes describe su actividad principal diaria como «Caminando, llevando algún peso, efectuando desplazamientos frecuentes», mientras que sólo el $2 \%$ considera que realiza tareas que requieren gran esfuerzo físico.

Por otro lado, el $21,67 \%$ de las personas participantes refiere estar de pie la mayora parte del día sin efectuar grandes desplazamientos o esfuerzos, y el $17,33 \%$ considera que su actividad principal es estar sentado/a la mayor parte del día. Estos porcentajes implican que casi el $40 \%$ de estas personas podrían ser consideradas como "poco o nada activas». En cuanto a las diferencias de género, se aprecian porcentajes similares excepto en la opción de «Sentado la mayor parte del día», dónde los hombres registran un mayor porcentaje.

Casi el 49\% de las personas participantes considera que su nivel de actividad física doméstica es «Medio», el 30,3\% lo percibe como «Alto», y el 14,3\% lo califica de «Bajo». En la Tabla 2 se muestra que las mujeres realizan mayor nivel de actividad doméstica que los hombres $(\mathrm{p}<0,005)$. El nivel de actividad físca doméstica está correlacionado negativamente con la edad $(\mathrm{p}<0,05)$ y positivamente con la condición física percibida $(\mathrm{p}<0,001)$. 
Tabla 2. Nivel de actividad física doméstica por sexo

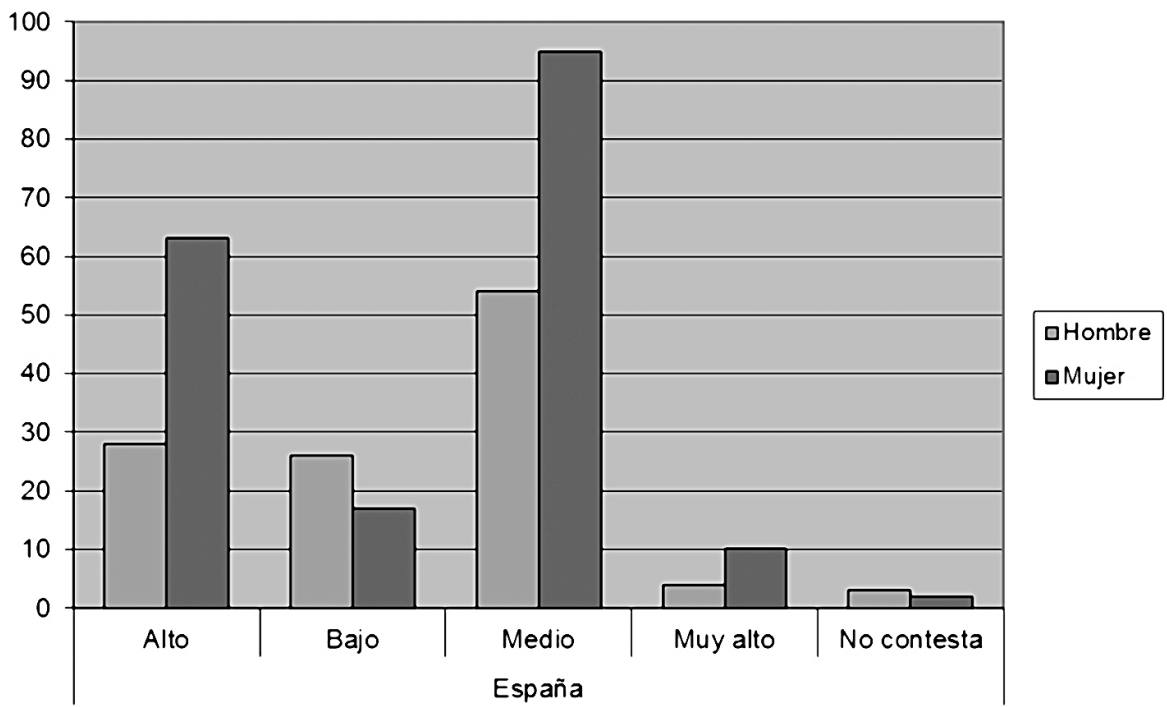

Respecto a la condición física percibida, el 48\% de las personas la considera como «Buena», el 41,7\% «Aceptable» y el 7,7\% Excelente. Tan sólo el 2,7\% de las personas percibe que su condición física es «Mala». No existen diferencias significativas entre el nivel de condición física percibida de las mujeres y de los hombres en la muestra, pero sí que disminuye significativamente con la edad $(\mathrm{p}<0,05)$.

El 28\% de las personas participantes en el estudio refiere realizar ejercicio físico entre 1 y 2 días a la semana en su tiempo libre, el 38,7\% realizan ejercicio físico entre 3 y 5 días, mientras que el 17,3\% realizan actividad física más de 5 días a la semana. El 15,3\% no realiza ningún día de la semana actividad física durante su tiempo libre. Los hombres realizan más días a la semana alguna actividad física que las mujeres $(p<0,05)$. El hacer mayor número de días actividad física está correlacionado positivamente con una mejor condición física percibida $(\mathrm{p}<0,001)$.

El 34\% de las personas indica que realiza más de 4 horas de actividad física a la semana, el 30,3\% realiza entre 2 y 4 horas, el 14\% realiza 2 horas, mientras que el 13\% realiza una hora. El 7,3\% no realizan ninguna hora de actividad física a la semana. Un mayor número de horas conllevan mejor 
condición física percibida $(\mathrm{p}<0,001)$. No existen diferencias significativas entre el número de horas que realizan las mujeres y las de los hombres.

El motivo principal por el que las personas encuestadas no realizan el ejercicio que deberían es «Por falta de voluntad», y el segundo motivo es «Porque prefiero realizar otro tipo de actividad de ocio (ir al cine, leer un libro, ver TV)». Las mujeres alegan como principal motivo preferir hacer otra cosa y en segundo lugar falta de tiempo.

Los hombres refieren mayor voluntad para realizar actividad física que las mujeres $(p<0,05)$. La voluntad para realizar ejercicio físico está correlacionada positivamente con la condición física percibida $(\mathrm{p}<0,001)$, con los días semanales de actividad física $(\mathrm{p}<0,001)$ y con las horas semanales $(\mathrm{p}<0,001)$.

\subsection{Actividad fisica y salud general}

Más del 70\% de las personas encuestadas consideró que su salud fue buena durante los doce últimos meses, el 10\% percibió su salud como excelente, el $17 \%$ opinó que su salud fue regular y menos del 1\% percibió su salud como mala. Por tanto, se podría decir que la salud de las personas participantes en general era buena. En cuanto a las diferencias de género, podemos observar que tanto hombres como mujeres consideraron mayoritariamente su salud buena, siendo este porcentaje mayor en los hombres con un $79,8 \%$ y las mujeres con un $66,3 \%$, aunque estas diferencias no fueron significativas. Esta salud percibida está correlacionada positivamente con la condición física percibida $(\mathrm{p}<0,001)$.

Encontramos diferencias significativas en la percepción de las personas encuestadas respecto a si su salud en ese momento les limitaba para realizar esfuerzos moderados (Tabla 3), siendo en mayor medida las mujeres quienes se quejaron de este hecho $(\mathrm{p}<0,05)$. Las personas que referían más limitaciones a este respecto fueron aquellos que realizaban menos actividad física doméstica $(p<0,001)$, tenían menos voluntad para realizar actividad física $(\mathrm{p}<0,001)$, tenían peor condición física percibida $(\mathrm{p}<0,001)$, y reaizaban menos días $(\mathrm{p}<0,001)$ y horas de actividad física semanal $(\mathrm{p}<0,001)$. 
Tabla 3. Percepción de que la salud haya limitado para realizar esfuerzos moderados

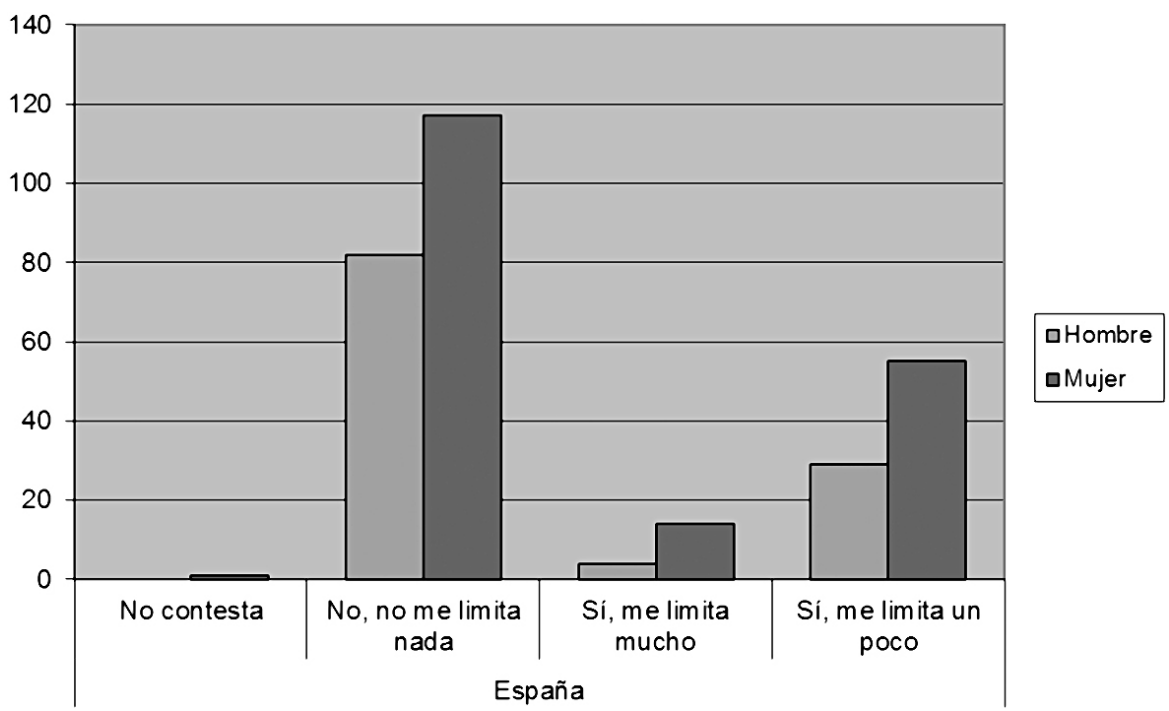

\subsection{Actividad física y salud emocional}

No se encuentran diferencias significativas entre la salud emocional percibida de las mujeres y de los hombres, excepto en que un mayor número de mujeres refieren perder el sueño por problemas emocionales $(\mathrm{p}<0,05)$.

Se encuentran correlaciones positivas entre la vitalidad y la actividad física doméstica $(p<0,001)$, la voluntad para realizar actividad física $(p<0,001)$, la condición física percibida ( $p<0,001)$, y la realización de más días $(p<0,001)$ y horas de actividad física semanal $(\mathrm{p}<0,001)$.

A su vez el estar más tranquilo y calmado está correlacionado positivamente con la actividad física doméstica $(p<0,01)$, la voluntad de realizar actividad física $(p<0,05)$ y la condición física percibida $(p<0,001)$. Por otro lado, las personas que en menor medida se sentían tristes y desanimadas eran aquellas que realizaban más actividad física doméstica $(\mathrm{p}<0,05)$ y tenían una mejor condición física percibida $(\mathrm{p}<0,001)$.

También correlaciona positivamente el grado en el cuál las personas encuestadas percibían que desenpeñaban un papel útil en la vida, con su actividad física doméstica $(p<0,01)$, la voluntad para realizar actividad física $(p<0,01)$, y su condición física percibida $(\mathrm{p}<0,005)$. Por otro lado, encontramos una 
correlación negativa entre la pérdida de confianza en uno mismo, y la actividad física doméstica $(\mathrm{p}<0,01)$ y la condición física percibida $(\mathrm{p}<0,001)$.

\subsection{Actividad física y memoria}

Cerca del $48 \%$ de las personas encuestadas considera que tiene problemas de memoria de algún tipo. Las mujeres se quejan más de perder el hilo de la conversación $(\mathrm{p}<0,05)$ y se encuentra una tendencia a que las mujeres tengan más problemas de orientación que no llega a ser significativa. Los hombres se quejan más de olvidar dónde han puesto cosas o de perder cosas $(\mathrm{p}<0,001)$, de problemas para encontrar objetos que necesitan $(p>0,001)$, y de olvidarse de cosas que tenían pensado hacer $(\mathrm{p}<0,01)$.

Las personas que refieren una mayor actividad física doméstica y una mejor condición física, tienen menos problemas a la hora de encontrar palabras cuando hablan o que parecen que la tienen «en la punta de la lengua» $(\mathrm{p}<0,01$; $\mathrm{p}<0,001)$, y a la hora de aprender nuevas habilidades $(\mathrm{p}<0,01 ; \mathrm{p}<0,005)$.

La actividad física doméstica, la condición física percibida, los días y las horas de actividad física semanal, están correlacionados negativamente con problemas para olvidar cosas que se tenían pensado hacer $(\mathrm{p}<0,005 ; \mathrm{p}<0,01$; $\mathrm{p}<0,001 ; \mathrm{p}<0,005 ; \mathrm{p}<0,001)$ y con problemas de desorientación $(\mathrm{p}<0,005$; $\mathrm{p}<0,05 ; \mathrm{p}<0,01 ; \mathrm{p}<0,05 ; \mathrm{p}<0,05)$.

Por último, aquellas personas que referían mayores niveles de actividad física doméstica y mejor condición física se quejaban de menos problemas para recordar nombres $(\mathrm{p}<0,01 ; \mathrm{p}<0,005)$ y recordar las caras de las personas $(\mathrm{p}<0,05 ; \mathrm{p}<0,05)$.

\section{Discusión}

El papel principal de las mujeres históricamente en muchas sociedades ha sido el de ser madres y cuidadoras de la familia, dejando en un segundo plano el tiempo de ocio y la actividad física. Este hecho ha ido cambiando en las últimas décadas, abriendo la posibilidad a las mujeres de incorporarse a la práctica de actividades lúdicas y deportivas ${ }^{17}$. Sin embargo, en España los hombres dedican más tiempo al día a deportes y actividades al aire libre que las mujeres en todos los tipos de hogar ${ }^{18}$.

Distintas corrientes feministas han abordado el papel del deporte en la vida social de las mujeres por la importancia social de esta institución. El

17. MERINO, Antonio. Op. Cit.

18. INE. Mujeres y Hombres en España. Madrid: INE-Ministerio de Sanidad, Servicios Sociales e Igualdad, 2012. 
feminismo liberal defiende el derecho individual de la mujer a participar en actividades deportivas. En cambio, las corrientes feministas radicales y sociales critican este enfoque individualista mientras paralelamente no exista una crítica al sistema capitalista y patriarcal imperante en muchas sociedades que mantiene el statu quo del hombre frente a la mujer. Para estas corrientes de pensamiento, el deporte contribuye a la exclusión de la mujer de la vida pública y a la subordinación de la mujer a la vida doméstica y la maternidad ${ }^{19}$.

En España, la incorporación de la mujer al deporte ha seguido un proceso de creación de una cultura deportiva femenina. Las mujeres en vez de imitar el modelo masculino del deporte, han creado uno propio ${ }^{20}$.

La esperanza de vida es mayor en las mujeres que en los hombres y ellas, cada vez más, están participando en actividades culturales y recreativas. En los programas de adultos mayores la participación femenina es de $7,9 \%$, demostrando su posibilidad para salir de su entorno de una forma organizada a través de grupos de actividad física o incluso que la mujer pueda ser más activa que los hombres a esta edad ${ }^{21}$.

En la presente investigación se han analizado las diferencias existentes entre hombres y mujeres mayores respecto a su participación en actividades físicas de ocio y domésticas y su condición física percibida. Estas variables se han correlacionado con la salud percibida general, procesos cognitivos y salud emocional.

\subsection{Niveles de actividad física y condición física}

Mayores niveles de actividad física en la edad adulta están asociados a una menor mortalidad ${ }^{22}$. Los resultados de nuestros datos con respecto a la actividad física en personas adultas reflejan que su actividad principal diaria es «activa» para casi un 60\%. Considerando que este grupo etario está mayoritariamente jubilado, estos resultados nos indican que probablemente el grupo activo ha logrado establecer un equilibrio en su vida, al tener intereses y poder explorar alternativas, que pueden ser gratificantes y significativas, al contrario

19. MESSNER, Michael, y SABO, Donald eds. Sport, men, and the gender order: Critical feminist perspectives. Champaign, IL: Human Kinetics Books, 1990.

20. PUIG, Nuria y SOLER, Susana. «Mujer y deporte en España: estado de la cuestión y propuesta interpretativa». Apunts Educación Física y Deportes, 76 (2004), pp. 71-78.

21. Merino, Antonio. Op. Cit.

22. Chen, L.-J., Fox, K.R., Ku, P.-W., Sun, W.-J., Chou, P. «Prospective Associations Between Household-, Work-, and Leisure-Based Physical Activity and All-Cause Mortality Among Older Taiwanese Adults». Asia Pac J Public Health 24 (2012), pp. 795-805. 
del grupo poco activo que el paso a la jubilación les puede provocar un sentimiento de inutilidad y desorientación ${ }^{23}$.

Los resultados del presente estudio muestran que las mujeres mayores realizan menos días a la semana actividad física que los hombres, aunque no existen diferencias significativas respecto al número de horas. En cambio, en un estudio llevado a cabo con personas mayores por Martínez del Castillo y colaboradores $^{24}$ et al. (2009), un mayor porcentaje de mujeres mayores realizaban actividad física y deportiva respeto a los hombres.

Otros estudios realizados en países de diferentes niveles de renta, sugieren que las mujeres realizan menos actividad física moderada y vigorosa que los hombres ${ }^{25}$, aunque cuando los estudios incluyen la actividad física doméstica estos niveles se equiparan, excepto para las actividades físicas vigorosas que las realizan en mayor medida los hombres ${ }^{26}$. La falta de actividad física vigorosa por parte de las mujeres puede ser debida a los estereotipos asociados a la actividad física y el deporte. Al ser el género una construcción social, las sociedades construyen las identidades de género atribuyendo a cada género unas características, atributos, apariencia física, vestimenta y comportamientos específicos ${ }^{27}$. Las actividades físico-deportivas de mayor intensidad están asociadas a los hombres mientras que a las mujeres se les reserva una actividad más moderada ${ }^{28}$. En este sentido, la falta de modelos femeninos en el deporte y de cobertura mediática ha ocasionado tanto una falta de participación como un abandono prematuro de la actividad deportiva en las mujeres. Los hombres han sido tradicionalmente apoyados emocionalmente para practicar deporte, reforzando su identidad de género, mientras ocurría lo contrario con las mujeres

23. Allan Natalia, Wachltz Daniela y VAldes Alicia. «Cambios en la Ocupación de los adultos mayores recientemente jubilados». Revista Chilena de terapia ocupacional, 5 (2005).

24. Martinez del Castillo, Jesús, Jimenez-Beatty, José, Gonzalez, María Dolores, Graupera, José Luis, Martin Rodriguez, María, Campos IzQuierdo, Antonio, DEL HIERRO, David. «Los hábitos de actividad física de las mujeres mayores en España». Revista internacional de Ciencias del deporte, 5 (14), (2009), pp. 81-93.

25. ReEd, S.B., CRESPO, C.J., HARVEY, W., ANDERSEN, R.E. «Social isolation and physical inactivity in older USadults: Results fromthe Third National Health and Nutrition Examination Survey». European Journal of Sport Sciences 11(5) (2011), pp. 347-353.

26. Azevedo Mario, Araujo Cora, Reichert Felipe, Siqueira Fernando, Silva Marcelo, HALLAL Pedro. «Gender differences in leisuretime physical activity». Int J Public Health 52 (2007), pp. 8-15.

27. ANDERSEN, Margaret L. Thinking about women - Sociological perspectives on sex and gender. New York, Maxmillan publishing company, 1993.

28. MESSNER, Michael y SABO, Donald, Op. cit., p. 11. 
Las mujeres en esta muestra realizan significativamente más actividad doméstica que los hombres, los cuales pasan más «sentados la mayor parte del tiempo». Estos resultados también coinciden con los de Lee ${ }^{29}$, en una muestra de 276 personas en Estados Unidos. Esto puede estar explicado por los roles que tanto los hombres como las mujeres tienen asumidos, estando todavía las labores de casa y el cuidado de la familia a cargo de las mujeres ${ }^{30} 31$. El Instituto de la Mujer realizó un estudio publicado en el 2007 donde constató que las mujeres en España siguen dedicando un tiempo significativamente mayor que los hombres al trabajo de casa ( 3 horas 35 minutos frente a 41 minutos) y al cuidado de la familia (1 hora 14 minutos frente a 38 minutos).

Una mayor actividad física doméstica está correlacionada con una mejor salud percibida en nuestra muestra y con un descenso de la mortalidad en estudios como el de Chen et al. ${ }^{32}$. No obstante, las mujeres deberían tener la oportunidad de tener tiempo libre y poder elegir su actividad física como parte de su tiempo de ocio y no verse obligadas a realizar las tareas domésticas debido a una imposición socio-cultural que pretende mantener el statu quo del hombre frente a la mujer ${ }^{33}$. Esta imposición se ha intentado justificar con la antigua idea de que las mujeres tienen mayor capacidad natural para cuidar de los niños y de la casa y que es algo inherente en ellas, es decir, han nacido para eso.

Por otro lado, la disminución de la actividad física tiene consecuencias especialmente perjudiciales para la mujer ya que sufre una mayor pérdida de masa ósea (osteopenia) con un alto porcentaje de osteoporosis ${ }^{34}$. La osteoporosis, unida al aumento en el número de caídas que se experimenta con la edad avanzada, provoca un gran número de fracturas en las mujeres ${ }^{35}$. Especialmente común es la fractura de cadera en mujeres.

A pesar de que los niveles de actividad física en la muestra son relativamente buenos, existe un alto porcentaje de personas que no realizan toda la actividad física que les gustaría. Las mujeres aluden a que prefieren hacer otra cosa y a la falta de tiempo. Sin embargo, los hombres hacen el ejercicio físico

29. LEE, Young Shin. «Gender differences in physical activity and walking among older adults». J Women Aging 17(1-2) (2005), pp. 55-70.

30. AlLan Natalia, WaChHOltz Daniela y VALDES Alicia, Op. Cit. p. 12.

31. CRESPO, María y LOPEZ, Javier. Cuidadoras y cuidadores: el efecto del género en el cuidado no profesional de los mayores. Madrid. IMSERSO, 2008.

32. CHEN, L.-J., Fox, K.R., Ku, P.-W., Sun, W.-J., y CHOU, P. «Prospective Associations Between Household-, Work-, and Leisure-Based Physical Activity and All-Cause Mortality Among Older Taiwanese Adults». Asia Pac J Public Health 24 (2012), pp. 795-805.

33. MeSSNER, Michael y SABO, Donald, Op. cit. p. 11.

34. Castelo, Camil, AYALA, Felix, y Brumel, Juan. Prevención y tratamiento de la osteoporosis. Genecologóa y Obstetricia 49 (4) (2003), pp. 248-258.

35. SEMI, Op. Cit. 
que desean en su tiempo libre en mayor porcentaje que las mujeres. De nuevo, esto puede ser debido a que las mujeres a pesar de su edad siguien ocupandose de las labores cotidianas de la casa ${ }^{36} 3738$, por lo que tienen menos tiempo libre que los hombres y menor libertad para emplear su tiempo de ocio.

Los niveles de actividad física se correlacionan de forma positiva con la condición física percibida. La condición física percibida por los participantes de este estudio es bastante buena. La segregación por género no revela diferencias significativas entre la percepción de los hombres y de las mujeres a este respecto. Otros estudios empleando medidas objetivas de condición física, como por ejemplo el estudio de Valentine et al. ${ }^{39}$, han encontrado un mayor nivel de condición física de los hombres respecto a las mujeres analizando el consumo máximo de oxígeno (VO2max) de una muestra de personas mayores. Esto puede ser debido a que los hombres realizan mayor tiempo de actividad física vigorosa semanal que las mujeres.

\subsection{Salud general y actividad física}

La percepción de la salud general es considerada como un indicador válido para predecir la salud futura y el riesgo de mortalidad en la personas mayores $^{40}$. La percepción general que tiene la muestra sobre su salud es bastante buena, con unos niveles relativamente buenos tanto en mujeres como hombres. Esta percepción correlaciona positivamente con la actividad física y la condición física percibida.

Esto puede ser debido a las características de la muestra. Los alumnos de la Universidad Permanente son en general personas con un nivel socioeconómico alto, así como un alto nivel de educación. Estos dos factores están correlacionados positivamente con la salud y, como sugieren Gonzalo y Pasarin ${ }^{41}$, con mayores niveles de actividad física que la población general.

36. Allan Natalia, Wachinoltz, Daniela y Valdes, Alicia. Op. Cit p. 12.

37. INSTITUTO DE LA MUJER. Usos del tiempo, Estereotipos, Valores y Actitudes. Madrid: Instituto de la Mujer-Ministerio de Trabajo y Asuntos Sociales, 2007.

38. CRESPO, María y LOPEZ, Javier, Op. Cit.

39. Valentine Rudy, Mcauley Edward, Vieira Victoria, Baynard Tracy, Hu Liang, Evans Ellen, y WooDS Jeffrey. «Sex differences in the relationship between obesity, C-reactive protein, physical activity, depression, sleep quality and fatigue in older adults». Brain Behav Immun 23 (2009), pp. 643-648.

40. LEE, Yunhwan. "The predictive value of self assessed general, physical, and mental health on functional decline and mortality in older adults». J Epidemiol Community Health 54 (2000), pp. 123-9.

41. GonZalo, Elena y PASARín, Maria. «La Salud de las personas mayores». Gac. Sanit 18 (1) (2004), pp. 69-80. 


\subsection{Actividad fisica y salud emocional}

Uno de los factores que influyen negativamente de una forma más decisiva en la salud percibida es la salud emocional ${ }^{42}$. No existen diferencias significativas entre hombres y mujeres respecto a su salud emocional, excepto que las mujeres refieren perder más el sueño por problemas emocionales que los hombres.

La actividad física puede mejorar el estado de ánimo y la autoestima, así como prevenir y mejorar el estrés, la ansiedad y la depresión ${ }^{43}$. Los resultados del presente estudio, sugieren efectos beneficiosos de la actividad física en la salud emocional de las personas mayores (vitalidad, estado de ánimo, sentirse útil en la vida) tanto en hombres como en mujeres. En las mujeres el riesgo de depresión es más elevado que en los hombres ${ }^{44}$. Por tanto, las mujeres se beneficiarían teóricamente más de realizar actividad física.

\subsection{Actividad física y memoria}

Casi la mitad de la muestra percibe tener problemas de memoria de algún tipo. Las mujeres refieren problemas para mantener el hilo de la conversación y una tendencia a desorientarse más que los hombres. Sin embargo, los hombres se quejan más de no poder encontrar objetos y de olvidar nombres. Aquellos que reportan realizar mayores niveles de actividad física y de condición física percibida, refieren menores problemas de memoria. Diferentes estudios demuestran que la actividad física puede mejorar los procesos cognitivos, incluido la memoria ${ }^{45}$. Una persona mayor que realiza regularmente actividad física presenta unas funciones cognitivas mejor conservadas, con una mayor superficie del hipocampo y una mejor memoria ${ }^{46}$. A su vez, la actividad física realizada de forma regular disminuye el riesgo de padecer enfermedades

42. AZPIAZU, Mercedes et al. «Factores asociados a mal estado de salud percibido o a mala calidad de vida en personas mayores de 65 años». Rev. Española Salud Pública 76 (6) (2002), pp. 683-699.

43. Chodzko-Zajko, Wojtek et al. American College of Sports Medicine position stand. Exercise and Physical Activity for Older Adults. Medicine \& Science in Sports \& Exercise 41(7) (2009).

44. Bergdahl E, Allard P, Gustafson Y. «Gender differences in depression among the very old». International Psychogeriatrics 19(6) (2007), pp. 1125-1140.

45. TAYLOR et al. «Physical activity and older adults: a review of health benefits and the effectiveness of interventions». Journal of Sports Sciences 22(8) (2004), pp. 703-725.

46. ERICKSON, Kirk et al. «Exercise training increases size of hippocampus and improves memory». Proceedings of the National Academy of Sciences of the United States of America 108 (7) (2011), pp. 3017-3022. 
neurodegenerativas asociadas con el envejecimiento, como la enfermedad de Alzheimer ${ }^{47}$.

Aparentemente las mujeres mayores experimentan mayores beneficios a nivel cognitivo por realizar actividad física que los hombres ${ }^{48}$. El efecto protector del estrógeno es una de las posibles explicaciones de estas diferencias ${ }^{49}$.

\section{Limitaciones del estudio}

Los resultados de este estudio están condicionados por las características peculiares de la muestra, compuesta por estudiantes de la Universidad Permanente de la Universidad de Alicante. Otra limitación del estudio es que se basa en un análisis de percepción y no en medidas objetivas. Sería interesante comparar el análisis de percepción con métodos objetivos. En el caso de los niveles de actividad física el uso de acelerómetros sería de gran utilidad. A su vez, sería interesante realizar un análisis en profundidad, empleando métodos cualitativos, sobre la participación de la mujer mayor en actividades físicas y deportivas, detectando barreras y facilitadores a la práctica así como factores de adherencia al ejercicio.

\section{Conclusión}

La actividad física desempeña un papel fundamental en el proceso de envejecimiento saludable, previniendo enfermedades, retrasando la dependencia y mejorando la calidad de vida de las personas mayores.

La mujer mayor suele realizar menos actividad física que el hombre, especialmente vigorosa, siendo la falta de tiempo uno de los principales motivos que alegan para no realizar toda la actividad física que les gustaría. Sin embargo, realizan más actividad doméstica que los hombres. Este hecho pone de manifiesto las barreras que todavía encuentran las mujeres a la hora de disponer de su tiempo de ocio ya que están al cargo de las tareas domésticas y el cuidado familiar en mayor medida que los hombres. A su vez, la falta de modelos deportivos femeninos y de cobertura mediática ha podido ocasionar tanto una falta de participación como un abandono prematuro de la actividad deportiva en las mujeres.

47. SCARMEAS, Nikolaos et al, «Physical Activity, Diet, and Risk of Alzheimer Disease». Journal of the American Medical Association 302 (6) (2009).

48. Colcombe, Stanley y Kramer, Arthur. «Fitness effects on the cognitive function of older adults: a meta-analytic study». Psychol Sci 14 (2003), pp. 125-30.

49. Ibidem. 
La percepción de la salud general se puede emplear como indicador para predecir la salud futura y el riesgo de mortalidad en personas mayores. Esta percepción de la salud está correlacionada positivamente con la cantidad de actividad física que se hace a la semana y con la condición física percibida. Respecto a la salud emocional, las mujeres de este estudio reportan perder más el sueño por problemas emocionales que los hombres. Por otro lado, cerca de la mitad de las personas mayores participantes en el estudio refieren algún tipo de problema de memoria. Las mujeres se quejan más de perder el hilo de la conversación y de una tendencia a desorientarse, mientras que los hombres de no poder encontrar objetos y de olvidar nombres.

Teniendo en cuenta que la mujer es más vulnerable a ciertos procesos físicos asociados con la edad como la osteoporosis, o emocionales como la depresión, y que se puede beneficiar más de la práctica de la actividad física como en la mejora de la memoria, es urgente poner en marcha políticas y estrategias encaminadas a la promoción de la actividad física en mujeres mayores.

\section{Referencias Bibliográficas}

Allan, Natalia WachHoltz, Daniela y Valdes, Alicia. «Cambios en la Ocupación de los adultos mayores recientemente jubilados». Revista Chilena de terapia ocupacional 5 (2005).

ANDERSEN, Margaret L. Thinking about women - Sociological perspectives on sex and gender. New York, Maxmillan publishing company, 1993.

ANDERSON, Bonnie y ZIUSSER, Judith. Historias de las mujeres: una historia propia. Barcelona, Crítica, 2003.

Azevedo Mario, Araujo Cora, Reichert Felipe, Siqueira Fernando, Silva Marcelo y HALLAL Pedro. «Gender differences in leisure time physical activity». Int J Public Health 52 (2007), pp. 8-15.

AZPIAZU, Mercedes et al. «Factores asociados a mal estado de salud percibido o a mala calidad de vida en personas mayores de 65 años». Revista Española de Salud Pública 76 (6) (2002), pp. 683-699.

BERNSTEIN, Alina. «Is it time for a victory lap? Changes in the media coverage of women in sport». International Review for the Sociology of Sport 37, (2002), pp. 415-428.

Bergdahl E., Allard, P. y Gustafson, Y. «Gender differences in depression among the very old». International Psychogeriatrics 19(6) (2007), pp.1125-1140.

Castelo, Camil., Ayala, Felix., y Brümel, Juan. «Prevención y tratamiento de la osteoporosis». Genecologóa y Obstetricia 49 (4) (2003), pp. 248-258.

Chen, L.-J., Fox, K.R., Ku, P.-W., Sun, W.-J., ChOu, P. «Prospective Associations Between Household, Work, and Leisure-Based Physical Activity and 
All-Cause Mortality Among Older Taiwanese Adults». Asia Pac J Public Health 24 (2012), pp. 795-805.

Chodzko-Zajko, Wojtek et al. «American College of Sports Medicine position stand. Exercise and Physical Activity for Older Adults». Medicine E Science in Sports E Exercise, 41(7), (2009).

COlCOMBE, Starley y KRAmer, Arthur. "Fitness effects on the cognitive function of older adults: a meta-analytic study». Psychol Sci 14 (2003), pp. 125-130.

CRESPO, María y LóPEZ, Javier. Cuidadoras y cuidadores: el efecto del género en el cuidado no profesional de los mayores. Madrid. IMSERSO, 2008.

EHLE - Empowering Health Learning for the Elderly. Funding: 134023 - LLP - 1 2007 -1 - IT - GRUNDTVIG-GMP <http://www.ehle-project.eu/>, consultado el 20-05-2013.

ERICKON, Kirk, VOSS, Michelle, PraKash, Ruchica, et al. «Exercise training increases size of hippocampus and improves memory». Proceedings of the National Academy of Sciences of the United States of America 108 (7) (2011), pp. 3017-3022.

GARCIA FERRANDO, Manuel. Aspectos sociales del deporte: una reflexión sociológica. Madrid, Alianza Editorial, 1990.

Gonzalo, Elena. \& PASARIN, María. «La Salud de las personas mayores». Gac. Sanit 18 (1) (2004), pp. 69-80.

INE Mujeres y Hombres en España. Madrid: INE-Ministerio de Sanidad, Servicios Sociales e Igualdad, 2012.

INSTITUTO DE LA MUJER. Usos del tiempo, Estereotipos, Valores y Actitudes. Madrid: Instituto de la Mujer-Ministerio de Trabajo y Asuntos Sociales, 2007.

LEE, Yunhwan. «The predictive value of self assessed general, physical, and mental health on functional decline and mortality in older adults». J Epidemiol Community Health 54 (2000), pp. 123-9.

LEE, Young Shin. «Gender differences in physical activity and walking among older adults». J Women Aging 17(1-2) (2005), pp. 55-70.

Lehr, Ursula y Thomae, Hans. Psicología de la senectud: proceso y aprendizaje del envejecimiento. Barcelona, Herder, 2003.

LÓPEZ, Cristina y AlvariÑas, Mirian. «Análisis muestrales desde una perspectiva de género en revistas de investigación de Ciencias de la Actividad Física y del Deporte españolas». Apunts: Educación física y deportes 106 (2011), pp. 62-70.

Martínez del Castillo, Jesús, JimÉnez-Beatty, José, GonZÁlez, María Dolores, Graupera, José Luis, Martín Rodríguez, María, Campos IzQuierdo, Antonio, Del Hierro, David. «Los hábitos de actividad física de las mujeres mayores en España». Revista internacional de Ciencias del deporte 5 (14), (2009), pp. 81-93. 
MERINO, Antonio. Mujer y deporte. Fórmula de integración en la sociedad actual. Motivaciones y necesidades específicas de la mujer en la realización de la práctica físico-deportiva. Diputación provincial de Jaén, 2007. <ttp://issuu.com/amerinomandly/docs/integracion_20social_20act_20fisica_20mujer $>$, consultado el 15-10-2012.

MESSNER, Michael y SABO, Donald (eds). Sport, men, and the gender order: Critical feminist perspectives. Champaign, IL: Human Kinetics Books, 1990.

MEAD, Margaret. Sexo y temperamento en tres sociedades primitivas. Barcelona: Paidós Ibérica, 2006.

MINISTERIO DE ASUNTOS SOCIALES. Mujer y deporte. Barcelona: Ministerio de Asuntos Sociales, 1990.

ORGANIZACIÓN MUNDIAL DE LA SALUD. Health and Development Through Physical Activity and Sport. <http://whqlibdoc.who.int/hq/2003/WHONMH_ NPH_PAH_03.2.pdf>, consultado el 20-03-2012.

ORGANIZACIÓN MUNDIAL DE LA SALUD. Recomendaciones mundiales sobre actividad física para la salud. <http://www.who.int/dietphysicalactivity/publications/9789241599979/es/index.html>, consultado el 20-03-2012.

PUIG, Nuria y SolER, Susana. «Mujer y deporte en España: estado de la cuestión y propuesta interpretativa». Apunts Educación Física y Deportes 76, (2004), pp. 71-78.

Reed, S.B., Crespo, C.J., Harvey, W., Andersen, R.E. «Social isolation and physical inactivity in older USadults: Results fromthe Third National Health and Nutrition Examination Survey». European Journal of Sport Sciences 11(5) (2011), pp. 347-353.

SCARMEAS, Nikolaos et al. «Physical Activity, Diet, and Risk of Alzheimer Disease» Journal of the American Medical Association 302 (6) (2009).

SEMI. Protocolos Osteoporosis. Sociedad Española de Medicina Interna, 2004.

TAYLOR et al. Physical activity and older adults: a review of health benefits and the effectiveness of interventions». Journal of Sports Sciences 22(8) (2004), pp. 703-725.

VAlentine Rudy, Mcauley Edward, Vieira Victoria, Baynard Tracy, Hu Liang, EVANS Ellen, WoODS Jeffrey. «Sex differences in the relationship between obesity, C-reactive protein, physical activity, depression, sleep quality and fatigue in older adults». Brain Behav Immun 23 (2009), pp. 643-648. 